\title{
Morbidity and Mortality After Benign Prostatic Hyperplasia Surgery: Data from the American College of Surgeons National Surgical Quality Improvement Program
}

\author{
Naeem Bhojani, MD, ${ }^{*}$ Giorgio Gandaglia, MD,, Akshay Sood, MD, ${ }^{2,3}$ Arun Rai, ${ }^{3,4}$ \\ Daniel Pucheril, MD, ${ }^{2}$ Steven L. Chang, MD, ${ }^{3}$ Pierre I. Karakiewicz, MD, Mani Menon, MD, ${ }^{2}$ \\ Kola Olugbade, Jr., ${ }^{3,5}$ Nedim Ruhotina, MD, ${ }^{3}$ Jesse D. Sammon, DO, ${ }^{2}$ Shyam Sukumar, MD, ${ }^{2}$ \\ Maxine Sun, BSc, Khurshid R. Ghani, BSc (Hons), MS, MRCS, ${ }^{5}$ Marianne Schmid, MD, ${ }^{3}$ Briony Varda, MD, ${ }^{3}$ \\ Adam S. Kibel, MD, Kevin C. Zorn, MD, and Quoc-Dien Trinh, MD ${ }^{3}$
}

\begin{abstract}
Background and Purpose: With the aging population, it is becoming increasingly important to identify patients at risk for postsurgical complications who might be more suited for conservative treatment. We sought to identify predictors of morbidity after surgical treatment of benign prostatic hyperplasia (BPH) using a large national contemporary population-based cohort.

Methods: Relying on the American College of Surgeons National Surgical-Quality Improvement Program (ACS-NSQIP; 2006-2011) database, we evaluated outcomes after transurethral resection of the prostate (TURP), laser vaporization of the prostate (LVP), and laser enucleation of the prostate (LEP). Outcomes included blood-transfusion rates, length of stay, complications, reintervention rates, and perioperative mortality. Multivariable logistic-regression analysis evaluated the predictors of perioperative morbidity and mortality. Results: Overall, 4794 (65.2\%), 2439 (33.1\%), and 126 (1.7\%) patients underwent TURP, LVP, and LEP, respectively. No significant difference in overall complications $(P=0.3)$ or perioperative mortality $(P=0.5)$ between the three surgical groups was found. LVP was found to be associated with decreased blood transfusions (odds ratio $[\mathrm{OR}]=0.21 ; P=0.001)$, length of stay $(\mathrm{OR}=0.12 ; P<0.001)$ and reintervention rates $(\mathrm{OR}=0.63$; $P=0.02)$. LEP was found to be associated with decreased prolonged length of stay $(\mathrm{OR}=0.35 ; P=0.01)$. Men with advanced age at surgery and non-Caucasians were at increased risk of morbidity and mortality. In contrast, normal preoperative albumin and higher preoperative hematocrit $(>30 \%)$ levels were the only predictors of lower overall complications and perioperative mortality.

Conclusions: All three surgical modalities for BPH management were found to be safe. Advanced age and nonCaucasian race were independent predictors of adverse outcomes after BPH surgery. In patients with these attributes, conservative treatment might be a reasonable alternative. Also, preoperative hematocrit and albumin levels represent reliable predictors of adverse outcomes, suggesting that these markers should be evaluated before BPH surgery.
\end{abstract}

\section{Introduction}

W ith THE aging U.S. population, a major concern is the increasing prevalence of urologic conditions in the elderly. The prevalence of lower urinary tract symptoms
(LUTS) increases significantly with age, increasing from $13 \%$ to $14 \%$ in the fifth decade of life to $28 \%$ to $43 \%$ after age $60{ }^{1-4}$ In addition, looking specifically at urinary retention (UR), a recent population-based study demonstrated that there has been a significant increase in UR and the need for

\footnotetext{
${ }^{1}$ Cancer Prognostics and Health Outcomes Unit, Centre Hospitalier de l'Université de Montréal, Montreal, Quebec, Canada.

${ }^{2}$ Vattikuti Urology Institute, Henry Ford Health System, Detroit, Michigan.

${ }^{3}$ Division of Urologic Surgery and Center for Surgery and Public Health, Brigham and Women's Hospital, Harvard Medical School, Boston, Massachusetts.

${ }^{4}$ Boston University School of Medicine, Boston, Massachusetts.

${ }^{5}$ University of Michigan Medical School, Ann Arbor, Michigan.

*Both authors contributed equally to this article.
} 
emergent urinary catheterizations from 2006 to $2009 .^{5} \mathrm{Si}$ milarly, Groves and associates ${ }^{6}$ found an increase in acute UR in men with benign prostatic hyperplasia (BPH). These trends suggest a growing demographic of men necessitating surgical intervention for BPH.

Transurethral resection of the prostate (TURP) has long been considered the primary surgical option for management of BPH in small to moderately sized prostate glands, with numerous studies demonstrating improvement in IPSS (International Prostate Symptom Score) and urinary flow in patients post-TURP. ${ }^{7,8}$ TURP can be associated with significant morbidity and even mortality, however. ${ }^{9}$ Complication and surgical revision rates increase with growing prostate size. ${ }^{10}$ Thus, newer endoscopic therapies have been developed over the past decade. ${ }^{11}$ Unfortunately, the long-term data on the efficacy/complications are not available for all these newer therapies, with holmium laser enucleation of the prostate (HoLEP) being the only modality to have been evaluated rigorously. A study by Krambeck and colleagues ${ }^{12}$ looking at more than 1000 HoLEPs demonstrated short-term ( $<6$ months), intermediate term (6-12 months), and longterm ( $>12$ months) mean IPSS of $8.7,5.9$, and 5.3, and maximum urinary flow of $17.9,19.5$, and $22.7 \mathrm{~mL} / \mathrm{sec}$, respectively. As well, the recent publication by Elmansy and coworkers $^{13}$ on their 10-year experience with HoLEP with a mean follow-up of more than 5 years reiterated these results.

Given the aging population (frequently with multiple comorbidities) and the lack of complication data on all the newer techniques, it is important to identify patients who may be at risk of developing complications from surgical intervention and who may be better served with conservative treatment. Therefore, we sought to identify the predictors of morbidity after surgical treatment of BPH using a large national contemporary population-based cohort of patients. We hypothesized that newer treatment modalities may have a protective effect on complication frequency for men otherwise at increased risk of complications.

\section{Methods}

\section{Population source}

The current study relied on the American College of Surgeons National Surgical Quality Improvement Program (NSQIP) database. Validated data from patients' medical charts allows quantification of 30-day, risk-adjusted surgical outcomes, including postdischarge, when nearly $50 \%$ of complications occur. Trained surgical clinical reviewers prospectively collect the data. In 2011, the ACS-NSQIP included data from 315 participating sites and more than 442,149 cases.

\section{Study population}

Overall, 7,359 men $\geq 18$ years of age undergoing a surgical procedure for BPH including TURP (Current Procedural Terminology [CPT] codes: 52601 and 52630), laser vaporization of the prostate (LVP) (CPT code: 52648) or laser enucleation and morcellation of the prostate (CPT-code: 52649) between 2006 and 2011 were identified. For the purpose of this study, because it is not known which laser was used for which technique, patients who underwent vaporization will be categorized as LVP and those patients who underwent enucleation will be categorized as laser enucle- ation of the prostate (LEP). Multiple imputations were used for the analysis of patients with missing data $(n=4247)$. In particular, 51 patients had unknown body mass index (BMI), 23 patients had unknown operative time, and 4247 patients had unknown preoperative albumin levels.

\section{Covariates}

For each patient, age at time of surgery, race, smoking status, BMI, anesthesia, operative time, and baseline comorbidities including diabetes, hypertension, cardiovascular and "other" (baseline dyspnea, chronic obstructive pulmonary disease, peripheral vascular disease, claudication, esophageal varices, hemodialysis, history of cerebrovascular accident or transient ischemic attack, hemiplegia, baseline renal insufficiency [creatininem $>2 \mathrm{mg} / \mathrm{dL}$ ]) were evaluated. In addition, preoperative albumin and hematocrit levels were available.

\section{Outcomes}

Postoperative complications were classified under the following categories: Overall, cardiovascular (including postoperative cardiac arrest, myocardial infarction, or cerebrovascular accident), pulmonary (including pneumonia, need for postoperative reintubation, and ventilator support $>48$ hours), thromboembolic (including deep venous thrombosis and pulmonary embolism), sepsis/shock (including systemic sepsis and septic shock), renal (including acute renal failure and progressive renal insufficiency), urinary tract infection, and wound infection (including superficial, deep, and organ-space surgical site infections, as well as wound dehiscence). Additional outcomes assessed were the need for reintervention (within 30 days), blood transfusion, prolonged length of stay (pLOS) ( $\geq 2$ days), and perioperative mortality.

\section{Statistical analyses}

Descriptive statistics of categoric variables focused on frequencies and proportions. Means, medians, and interquartile ranges were reported for continuous variables.

Univariable logistic regression models tested the association between preoperative covariates (including the type of surgical procedure performed) and the rate of blood transfusions, pLOS, overall complications, and perioperative mortality rates. Multivariable logistic regression models including only predictors at univariable analyses were then generated.

All statistical tests were performed using the R statistical package (version 3.0.2), with a two-sided significance level set at $P<0.05$.

\section{Results \\ Baseline characteristics}

Overall, 7359 patients with BPH undergoing surgical treatment at one of the NSQIP participating hospitals between 2006 and 2011 were included. Of these, 4794 (65.2\%), $2439(33.1 \%)$, and $126(1.7 \%)$ patients underwent TURP, LVP, and LEP, respectively.

Table 1 displays the baseline characteristics of the cohort. When men were stratified according to the surgical procedure they underwent (TURP vs LVP vs LEP), statistically significant differences were observed with regard to the age at time of surgery $(P<0.001)$, race $(P<0.001)$, preoperative hematocrit 
Table 1. Descriptive Characteristics of 7359 Patients $\geq 18$ Years Old Undergoing Either Transurethral Resection of the Prostate or Tissue Vaporization or Enucleation for Management of Benign Prostatic Hyperplasia (National Surgical Quality Improvement Program Database 2006-2009)

\begin{tabular}{|c|c|c|c|c|c|}
\hline Variables & Overall (\%) & $T U R P(\%)$ & Tissue vaporization (\%) & Enucleation (\%) & $\mathrm{P}$ \\
\hline Number of patients $(\%)$ & $7359(100)$ & $4794(65.2)$ & $2439(33.1)$ & $126(1.7)$ & - \\
\hline Age (years, mean) & 71.8 & 72.2 & 71.0 & 69.0 & $<0.001$ \\
\hline \multicolumn{6}{|l|}{ Race; $n(\%)$} \\
\hline Caucasian & $5478(74.4)$ & 3448 (71.9) & $1935(79.3)$ & $95(75.4)$ & \multirow[t]{2}{*}{$<0.001$} \\
\hline Other & $1881(25.6)$ & $1346(28.1)$ & $504(20.7)$ & $31(24.6)$ & \\
\hline \multicolumn{6}{|l|}{ Smoking; $n(\%)$} \\
\hline Nonsmoker & $6565(89.2)$ & 4264 (88.9) & 2194 (89.9) & 107 (84.9) & \multirow[t]{2}{*}{0.125} \\
\hline Smoker & 794 (10.8) & $530(11.1)$ & $245(10.0)$ & $19(15.1)$ & \\
\hline \multicolumn{6}{|l|}{$\mathrm{BMI} ; n(\%)$} \\
\hline$<25$ & 2134 (29.0) & $1390(29.0)$ & 705 (28.9) & $39(31.0)$ & \multirow[t]{3}{*}{0.683} \\
\hline $25-30$ & $3062(41.6)$ & $1981(41.3)$ & $1024(42.0)$ & $57(45.2)$ & \\
\hline$>30$ & $2163(29.4)$ & $1423(29.7)$ & $710(29.1)$ & $30(23.8)$ & \\
\hline \multicolumn{6}{|l|}{ Preoperative comorbidities } \\
\hline Diabetes; $n(\%)$ & $1513(20.6)$ & $1018(21.2)$ & $471(19.3)$ & $24(19.0)$ & 0.146 \\
\hline Hypertension; $n(\%)$ & $4684(63.6)$ & $3083(64.3)$ & $1524(62.5)$ & $77(61.1)$ & 0.261 \\
\hline Cardiovascular comorbidities; $n(\%)$ & $1266(17.2)$ & $805(16.8)$ & $445(18.2)$ & $16(12.7)$ & 0.121 \\
\hline Other cedical comorbidities; $n(\%)$ & $1359(18.5)$ & $881(18.4)$ & $468(19.2)$ & $10(7.9)$ & 0.006 \\
\hline \multicolumn{6}{|l|}{ Hematocrit; $n(\%)$} \\
\hline$<30$ & $307(4.2)$ & $232(4.8)$ & $73(3.0)$ & $2(1.6)$ & \multirow[t]{3}{*}{0.001} \\
\hline $30-45$ & $5931(80.6)$ & $3850(80.3)$ & $1984(81.3)$ & $97(77.0)$ & \\
\hline$>45$ & $1121(15.2)$ & $712(14.9)$ & $382(15.7)$ & $27(21.4)$ & \\
\hline \multicolumn{6}{|l|}{ Anesthesia; $n(\%)$} \\
\hline General anesthesia & $1985(27.0)$ & $1534(32.0)$ & $433(17.8)$ & $18(14.3)$ & \multirow[t]{2}{*}{$<0.001$} \\
\hline Spinal anesthesia & $5374(73.0)$ & $3260(68.0)$ & $2006(82.2)$ & $108(85.7)$ & \\
\hline \multicolumn{6}{|l|}{ Preoperative albumin; $n(\%)$} \\
\hline$<2.5$ & $109(3.5)$ & $80(4.3)$ & $28(2.3)$ & $1(2.8)$ & \multirow[t]{3}{*}{$<0.001$} \\
\hline $2.5-3.5$ & $687(22.1)$ & $443(23.8)$ & $241(19.9)$ & $3(8.3)$ & \\
\hline$>3.5$ & $2316(74.4)$ & $1339(71.9)$ & $945(77.8)$ & $32(88.9)$ & \\
\hline Operative time (minutes, mean) & 56.9 & 54.7 & 57.4 & 139.4 & $<0.001$ \\
\hline
\end{tabular}

TURP $=$ transurethral resection of the prostate; $\mathrm{BMI}=$ body mass index.

value $(P=0.001)$, anesthetic type $(P<0.001)$, preoperative albumin level $(P<0.001)$, and operative time $(P<0.001)$. In particular, men undergoing LEP were significantly less likely to have a medical comorbidity classified as "other" when compared with men in the TURP or LVP cohorts. In addition, a larger percentage of men undergoing LEP had a preoperative hematocrit value $>45 \%$ and preoperative serum albumin level $>3.5 \mathrm{~g} / \mathrm{dL}$ compared with men undergoing TURP and LVP. The proportion of patients who underwent TURP under general anesthesia was significantly higher compared with

Table 2. Outcomes in 7359 Patients $\geq 18$ Years Old Undergoing Either Transurethral Resection of the Prostate or Tissue Vaporization or Enucleation for Management of Benign Prostatic Hyperplasia (National Surgical Quality Improvement Program Database 2006-2009)

\begin{tabular}{|c|c|c|c|c|c|}
\hline Variables & Overall (\%) & TURP (\%) & Tissue vaporization (\%) & Enucleation (\%) & $\mathrm{P}$ \\
\hline Number of patients $(\%)$ & $7359(100)$ & $4794(65.2)$ & $2439(33.1)$ & $126(1.7)$ & - \\
\hline Complications & & & & & \\
\hline Overall complications; $n(\%)$ & $472(6.4)$ & $312(6.5)$ & $156(6.4)$ & $4(3.2)$ & 0.321 \\
\hline Cardiovascular complications; $n(\%)$ & $21(0.3)$ & $16(0.3)$ & $5(0.2)$ & $0(0.0)$ & 0.520 \\
\hline Pulmonary complications; $n(\%)$ & $42(0.6)$ & $35(0.7)$ & $7(0.3)$ & $0(0.0)$ & 0.042 \\
\hline Thromboembolic complications; $n(\%)$ & $27(0.4)$ & $19(0.4)$ & $8(0.3)$ & $0(0.0)$ & 0.712 \\
\hline Sepsis/shock complications; $n(\%)$ & $69(0.9)$ & $52(1.1)$ & $17(0.7)$ & $0(0.0)$ & 0.147 \\
\hline Renal failure complications; $n(\%)$ & $23(0.3)$ & $18(0.4)$ & $5(0.2)$ & $0(0.0)$ & 0.385 \\
\hline UTI complications; $n(\%)$ & $368(5.0)$ & $234(4.9)$ & $130(5.3)$ & $4(3.2)$ & 0.453 \\
\hline Wound complications; $n(\%)$ & $10(0.1)$ & $6(0.1)$ & $4(0.2)$ & $0(0.0)$ & 0.838 \\
\hline Need for reintervention; $n(\%)$ & $138(1.9)$ & $104(2.2)$ & $32(1.3)$ & $2(1.6)$ & 0.038 \\
\hline Perioperative mortality; $n(\%)$ & $27(0.4)$ & $20(0.4)$ & $7(0.3)$ & $0(0.0)$ & 0.543 \\
\hline Blood transfusion; $n(\%)$ & $109(1.5)$ & $94(2.0)$ & $12(0.5)$ & $3(2.4)$ & $<0.001$ \\
\hline Prolonged length of stay; $n(\%)$ & $2122(28.8)$ & $1897(39.6)$ & $198(8.1)$ & $27(21.4)$ & $<0.001$ \\
\hline
\end{tabular}


those treated with LVP or LEP. Finally, patients undergoing LEP had significantly longer operative times.

\section{Unadjusted postoperative complications rates}

Table 2 depicts the rates of postoperative complications, blood transfusions, pLOS, reintervention, and perioperative mortality. There was no statistically significant difference in rates of overall complications among the three treatment groups $(P=0.3)$. Patients undergoing TURP, however, had significantly higher rates of pulmonary complications compared with other surgical modalities $(P=0.04)$. Urinary tract infections were by far the most frequent complication and comparable among surgical groups.

Patients undergoing LVP were less likely to need blood transfusions compared with patients treated with other surgical modalities $(P<0.001)$. Patients undergoing TURP were significantly more likely to need subsequent reintervention and to experience a pLOS $(P<0.04$ and $P<0.001$, respec- tively). Finally, no significant difference was observed in postoperative mortality between the three surgical modalities $(P=0.5)$.

\section{Uni- and multivariable logistic regression analyses}

Table 3 reports the predictors of morbidity and mortality after BPH surgery. In multivariate analysis, both age (odds ratio $[\mathrm{OR}]=1.03 ; P=0.001$ ) and non-Caucasian race $(\mathrm{OR}=1.55 ; P=0.006)$ were significant predictors of overall complications (Table 3A). In particular, the odds of experiencing overall complications increased by $3 \%$ as the age increased by 1 year $(P=0.001)$. In contrast, normal preoperative albumin levels $(>3.5 \mathrm{~g} / \mathrm{dL})$ were associated with a lower risk of experiencing overall complications $(\mathrm{OR}=0.41$; $P=0.002)$. Overweight patients (BMI 25-30) $(\mathrm{OR}=0.49$; $P=0.03$ ), patients with higher preoperative hematocrits $(30 \%-45 \%)(\mathrm{OR}=0.09 ; P<0.001)$, patients undergoing LVP $(\mathrm{OR}=0.21 ; P=0.001)$, and those with normal preoperative

Table 3A. Uni- and Multivariable Logistic Regression Analysis of Predictors of Complications in 7359 Patients Undergoing Either Transurethral Resection of the Prostate or Tissue Vaporization or Enucleation for Management of Benign Prostatic Hyperplasia (National Surgical Quality Improvement Program Database 2006-2009)

\begin{tabular}{|c|c|c|c|c|}
\hline & \multicolumn{2}{|c|}{ Univariable analyses } & \multicolumn{2}{|c|}{ Multivariable analyses } \\
\hline & Odds ratio & $\mathrm{P}$ value & Odds ratio & $\mathrm{P}$ value \\
\hline Age at procedure & $1.03(1.02-1.05)$ & 0.001 & $1.03(1.01-1.04)$ & 0.001 \\
\hline $\begin{array}{l}\text { Race } \\
\text { Caucasian } \\
\text { Other }\end{array}$ & $\begin{array}{c}1(\text { ref }) \\
1.60(1.31-1.95)\end{array}$ & 0.006 & $\begin{array}{c}1 \text { (Ref) } \\
1.55(1.13-2.13)\end{array}$ & 0.006 \\
\hline $\begin{array}{l}\text { Smoking } \\
\text { Nonsmoker } \\
\text { Smoker }\end{array}$ & $\begin{array}{c}1 \text { (Ref) } \\
0.98(0.72-1.32)\end{array}$ & 0.8 & - & \\
\hline $\begin{array}{l}\text { BMI } \\
<25 \\
25-30 \\
>30\end{array}$ & $\begin{array}{c}1 \text { (Ref) } \\
0.93(0.76-1.18) \\
0.96(0.75-1.22)\end{array}$ & $\begin{array}{l}0.5 \\
0.8\end{array}$ & - & \\
\hline $\begin{array}{l}\text { Properative comorbidities } \\
\text { Diabetes } \\
\text { Hypertension } \\
\text { Cardiovascular } \\
\text { Other }\end{array}$ & $\begin{array}{l}1.32(1.07-1.65) \\
1.25(1.03-1.52) \\
1.33(1.06-1.68) \\
1.65(1.33-2.04)\end{array}$ & $\begin{array}{c}0.01 \\
0.02 \\
0.01 \\
<0.001\end{array}$ & $\begin{array}{l}1.32(0.98-1.85) \\
1.08(0.78-1.48) \\
1.08(0.77-1.53) \\
1.35(0.99-1.84)\end{array}$ & $\begin{array}{l}0.06 \\
0.6 \\
0.6 \\
0.07\end{array}$ \\
\hline $\begin{array}{l}\text { Hematocrit }(\%) \\
<30 \\
30-45 \\
>45\end{array}$ & $\begin{array}{c}1(\text { Ref }) \\
0.36(0.26-0.50) \\
0.26(0.17-0.39)\end{array}$ & $\begin{array}{l}0.001 \\
0.001\end{array}$ & $\begin{array}{c}1(\text { Ref }) \\
0.72(0.46-1.14) \\
0.58(0.30-1.11)\end{array}$ & $\begin{array}{l}0.1 \\
0.2\end{array}$ \\
\hline $\begin{array}{l}\text { Anesthesia } \\
\text { General } \\
\text { Spinal }\end{array}$ & $\begin{array}{c}1 \text { (Ref) } \\
1.15(0.91-1.37)\end{array}$ & 0.3 & - & \\
\hline $\begin{array}{l}\text { Surgery } \\
\text { TURP } \\
\text { Vaporization } \\
\text { Enucleation }\end{array}$ & $\begin{aligned} 1 & (\text { Ref }) \\
0.98 & (0.80-1.19) \\
0.74 & (0.17-1.28)\end{aligned}$ & $\begin{array}{l}0.8 \\
0.1\end{array}$ & $\begin{array}{c}1(\text { Ref }) \\
0.99(0.75-1.33) \\
1.38(0.40-4.73)\end{array}$ & $\begin{array}{l}0.9 \\
0.6\end{array}$ \\
\hline $\begin{array}{l}\text { Preoperative albumin }(\mathrm{g} / \mathrm{dL}) \\
<2.5 \\
2.5-3.5 \\
>3.5 \\
\text { Operative time }\end{array}$ & $\begin{aligned} & 1(\text { Ref }) \\
& 0.65(0.37-1.14) \\
& 0.19(0.12-0.33) \\
& 1.00(0.99-1.00)\end{aligned}$ & $\begin{array}{l}0.1 \\
0.001 \\
0.9\end{array}$ & $\begin{array}{c}1 \text { (Ref) } \\
0.72(0.40-1.28) \\
0.41(0.23-0.73) \\
\quad-\end{array}$ & $\begin{array}{l}0.2 \\
0.002\end{array}$ \\
\hline
\end{tabular}

$\mathrm{BMI}=$ body mass index; TURP $=$ transurethral resection of the prostate. 
Table 3B. Uni- and Multivariable Logistic Regression Analysis of Predictors of Need for Blood Transfusions in 7359 Patients Undergoing Either Transurethral Resection of the Prostate or Tissue Vaporization or Enucleation for Management of Benign Prostatic Hyperplasia

(National Surgical Quality Improvement Program Database 2006-2009)

\begin{tabular}{|c|c|c|c|c|}
\hline & \multicolumn{2}{|c|}{ Univariable analyses } & \multicolumn{2}{|c|}{ Multivariable analyses } \\
\hline & Odds ratio & $\mathrm{P}$ value & Odds ratio & $\mathrm{P}$ value \\
\hline Age at procedure & $1.06(1.04-1.08)$ & $<0.001$ & $1.02(0.98-1.05)$ & 0.2 \\
\hline $\begin{array}{l}\text { Race } \\
\text { Caucasian } \\
\text { Other }\end{array}$ & $\begin{array}{c}1 \text { (Ref) } \\
1.99(1.35-2.94)\end{array}$ & 0.001 & $\begin{array}{c}1 \text { (Ref) } \\
1.31(0.71-2.42)\end{array}$ & 0.4 \\
\hline $\begin{array}{l}\text { Smoking } \\
\text { Nonsmoker } \\
\text { Smoker }\end{array}$ & $\begin{array}{l}1 \text { (Ref) } \\
0.83(0.44-1.60)\end{array}$ & 0.5 & - & \\
\hline $\begin{array}{l}\text { BMI } \\
<25 \\
25-30 \\
>30\end{array}$ & $\begin{array}{l}1 \text { (Ref) } \\
0.37(0.24-0.58) \\
0.35(0.21-0.59)\end{array}$ & $\begin{array}{l}0.001 \\
0.001\end{array}$ & $\begin{array}{c}1 \text { (Ref) } \\
0.49(0.25-0.96) \\
0.49(0.23-1.07)\end{array}$ & $\begin{array}{l}0.03 \\
0.1\end{array}$ \\
\hline $\begin{array}{l}\text { Preoperative comorbidities } \\
\text { Diabetes } \\
\text { Hypertension } \\
\text { Cardiovascular } \\
\text { Other }\end{array}$ & $\begin{array}{l}1.09(0.69-1.75) \\
1.83(1.18-2.86) \\
1.22(0.76-1.96) \\
1.61(1.05-2.47)\end{array}$ & $\begin{array}{l}0.7 \\
0.01 \\
0.4 \\
0.03\end{array}$ & $\begin{array}{c}1.09(0 . \overline{57}-2.07) \\
\overline{-} \\
1.03(0.53-2.01)\end{array}$ & $\begin{array}{l}0.4 \\
0.8 \\
0.9 \\
0.03\end{array}$ \\
\hline $\begin{array}{l}\text { Hematocrit }(\%) \\
<30 \\
30-45 \\
>45\end{array}$ & $\begin{array}{c}1 \text { (Ref) } \\
0.03(0.02-0.05) \\
0.01(0.01-0.03)\end{array}$ & $\begin{array}{l}<0.001 \\
<0.001\end{array}$ & $\begin{aligned} & 1 \text { (Ref) } \\
& 0.09(0.04-0.15) \\
& 0.00(0.00-0.00)\end{aligned}$ & $\begin{array}{c}<0.001 \\
0.9\end{array}$ \\
\hline $\begin{array}{l}\text { Anesthesia } \\
\text { General } \\
\text { Spinal }\end{array}$ & $\begin{array}{c}1 \text { (Ref) } \\
1.07(0.71-1.64)\end{array}$ & 0.7 & - & \\
\hline $\begin{array}{l}\text { Surgery } \\
\text { TURP } \\
\text { Vaporization } \\
\text { Enucleation }\end{array}$ & $\begin{array}{l}1 \text { (Ref) } \\
0.24(0.13-0.45) \\
1.22(0.38-3.90)\end{array}$ & $\begin{array}{l}0.001 \\
0.7\end{array}$ & $\begin{array}{c}1 \text { (Ref) } \\
0.21(0.09-0.51) \\
1.03(0.10-10.44)\end{array}$ & $\begin{array}{l}0.001 \\
0.9\end{array}$ \\
\hline $\begin{array}{l}\text { Preoperative albumin }(\mathrm{g} / \mathrm{dL}) \\
<2.5 \\
2.5-3.5 \\
>3.5 \\
\text { Operative time }\end{array}$ & $\begin{array}{c}1 \text { (Ref) } \\
0.30(0.15-0.63) \\
0.08(0.03-0.16) \\
1.01(1.01-1.02)\end{array}$ & $\begin{array}{l}0.001 \\
0.001 \\
0.001\end{array}$ & $\begin{array}{c}1(\text { Ref }) \\
1.00(0.96-1.01) \\
0.56(0.25-0.65) \\
1.00(1.00-1.01)\end{array}$ & $\begin{array}{l}0.1 \\
0.02 \\
0.06\end{array}$ \\
\hline
\end{tabular}

$\mathrm{BMI}=$ body mass index; TURP $=$ transurethral resection of the prostate.

albumin levels $(\mathrm{OR}=0.56 ; P=0.02)$ were less likely to need postoperative blood transfusion (Table $3 \mathrm{~B})$.

Increasing age $(\mathrm{OR}=1.01 ; P<0.001)$, diabetes $(\mathrm{OR}=1.23$; $P=0.03)$, presence of other comorbidities $(\mathrm{OR}=1.35 ; P=0.01)$ and longer operative time $(\mathrm{OR}=1.01 ; P<0.001)$ were all associated with a pLOS (Table 3C). Moderately/severely obese patients $(\mathrm{BMI}>30)(\mathrm{OR}=0.75 ; P=0.02)$, patients with higher preoperative hematocrits $(\geq 30 \%)(\mathrm{OR}=0.30 ; P<0.001)$, those patients receiving a spinal anesthetic $(\mathrm{OR}=0.67 ; P<0001)$, patients undergoing $\mathrm{LVP}(\mathrm{OR}=0.12 ; P<0.001)$ or $\mathrm{LEP}$ $(\mathrm{OR}=0.35 ; P=0.01)$, and those patients with preoperative albumin levels between 2.5 and $3.5 \mathrm{~g} / \mathrm{dL}(\mathrm{OR}=0.44 ; P=$ $0.001)$ and $>3.5 \mathrm{~g} / \mathrm{dL}(\mathrm{OR}=0.27 ; P<0.001)$ were all associated with a decreased rate of pLOS.

Multivariable analysis for predictors of perioperative mortality demonstrated that both age $(\mathrm{OR}=1.10 ; P=0.01)$ and other comorbidities $(\mathrm{OR}=3.13 ; P=0.04)$ were associated with increased perioperative mortality. In contrast, higher preoperative hematocrit $(30 \%-45 \%)(\mathrm{OR}=0.21 ; P=$
$0.01)$ and preoperative albumin levels $>3.5 \mathrm{~g} / \mathrm{dL}(\mathrm{OR}=0.13$; $P=0.01)$ were the only significant predictors of lower perioperative mortality (Table 3D). Examining reintervention rates, the only predictor of decreased rate of reintervention was patients undergoing $\operatorname{LVP}(\mathrm{OR}=0.63 ; P=0.02$; Table 3E).

\section{Discussion}

LUTS and UR are common in the aging population. ${ }^{14,15}$ An epidemiology of LUTS study by Coyne and coworkers ${ }^{15}$ in 2008 estimated the prevalence of at least one LUTS as defined by the International Continence Study for at least "sometimes" and "often" at $72.3 \%$ and $47.9 \%$, respectively. Furthermore, with the rightward shift in the population demographics, more men will be presenting with LUTS and/or UR. In a recent article, Roghmann and colleagues ${ }^{5}$ demonstrated that although the number of patients presenting to the emergency department with LUTS was stable between 2006 
Table 3C. Uni- and Multivariable Logistic Regression Analysis of Predictors of Prolonged Operative Stay in 7359 Patients Undergoing Either Transurethral Resection of the Prostate or Tissue Vaporization or Enucleation for Management of Benign Prostatic Hyperplasia (National Surgical Quality Improvement Program Database 2006-2009)

\begin{tabular}{|c|c|c|c|c|}
\hline & \multicolumn{2}{|c|}{ Univariable analyses } & \multicolumn{2}{|c|}{ Multivariable analyses } \\
\hline & Odds ratio & $\mathrm{P}$ value & Odds ratio & $\mathrm{P}$ value \\
\hline Age at procedure & $1.03(1.02-1.03)$ & 0.001 & $1.01(1.00-1.02)$ & 0.001 \\
\hline $\begin{array}{l}\text { Race } \\
\text { Caucasian } \\
\text { Other }\end{array}$ & $\begin{array}{c}1 \text { (Ref) } \\
1.49(1.34-1.67)\end{array}$ & 0.001 & $\begin{array}{c}1 \text { ( }(\text { Ref }) \\
0.98(0.78-1.22)\end{array}$ & 0.8 \\
\hline $\begin{array}{l}\text { Smoking } \\
\text { Nonsmoker } \\
\text { Smoker }\end{array}$ & $\begin{array}{c}1 \text { (Ref) } \\
1.00(0.86-1.18)\end{array}$ & 0.9 & - & \\
\hline $\begin{array}{l}\text { BMI } \\
<25 \\
25-30 \\
>30\end{array}$ & $\begin{array}{c}1 \text { (Ref) } \\
0.81(0.72-91) \\
0.73(0.64-0.84)\end{array}$ & $\begin{array}{l}0.001 \\
0.001\end{array}$ & $\begin{aligned} 1 & \text { (Ref) } \\
0.92 & (0.75-1.22) \\
0.75 & (0.59-0.96)\end{aligned}$ & $\begin{array}{l}0.4 \\
0.02\end{array}$ \\
\hline $\begin{array}{l}\text { Preoperative comorbidities } \\
\text { Diabetes } \\
\text { Hypertension } \\
\text { Cardiovascular } \\
\text { Other }\end{array}$ & $\begin{array}{l}1.29(1.14-1.46) \\
1.11(1.00-1.23) \\
1.15(1.01-1.32) \\
1.43(1.30-1.66)\end{array}$ & $\begin{array}{l}0.001 \\
0.04 \\
0.03 \\
0.001\end{array}$ & $\begin{array}{l}1.25(1.01-1.55) \\
1.00(0.83-1.22) \\
1.18(0.94-1.50) \\
1.35(1.09-1.68)\end{array}$ & $\begin{array}{l}0.03 \\
0.9 \\
0.1 \\
0.01\end{array}$ \\
\hline $\begin{array}{l}\text { Hematocrit }(\%) \\
<30 \\
30-45 \\
>45\end{array}$ & $\begin{array}{c}1(\text { Ref }) \\
0.29(0.17-0.28) \\
0.16(0.12-0.21)\end{array}$ & $\begin{array}{l}<0.001 \\
<0.001\end{array}$ & $\begin{array}{c}1 \text { (Ref) } \\
0.30(0.21-0.43) \\
0.29(0.19-0.45)\end{array}$ & $\begin{array}{l}<0.001 \\
<0.001\end{array}$ \\
\hline $\begin{array}{l}\text { Anesthesia } \\
\text { General } \\
\text { Spinal }\end{array}$ & $\begin{array}{c}1 \text { (Ref) } \\
1.79(1.61-2.00)\end{array}$ & $<0.001$ & $\begin{array}{c}1 \text { (Ref) } \\
0.67(0.55-0.82)\end{array}$ & $<0.001$ \\
\hline $\begin{array}{l}\text { Surgery } \\
\text { TURP } \\
\text { Vaporization } \\
\text { Enucleation }\end{array}$ & $\begin{array}{c}1(\text { Ref }) \\
0.14(0.11-0.16) \\
0.41(0.27-0.64)\end{array}$ & $\begin{array}{l}0.001 \\
0.001\end{array}$ & $\begin{aligned} 1 & (\text { Ref }) \\
0.12 & (0.10-0.15) \\
0.35 & (0.15-0.79)\end{aligned}$ & $\begin{array}{c}<0.001 \\
0.01\end{array}$ \\
\hline $\begin{array}{l}\text { Preoperative albumin }(\mathrm{g} / \mathrm{dL}) \\
<2.5 \\
2.5-3.5 \\
>3.5 \\
\text { Operative time }\end{array}$ & $\begin{array}{c}1 \text { (Ref) } \\
0.38(0.25-0.57) \\
0.18(0.12-0.27) \\
1.01(1.01-1.02)\end{array}$ & $\begin{array}{r}0.001 \\
<0.001 \\
<0.001\end{array}$ & $\begin{array}{c}1 \text { (Ref) } \\
0.44(0.27-0.71) \\
0.27(0.17-0.43) \\
1.01(1.00-1.01)\end{array}$ & $\begin{array}{r}0.001 \\
<0.001 \\
<0.001\end{array}$ \\
\hline
\end{tabular}

$\mathrm{BMI}=$ body mass index; TURP $=$ transurethral resection of the prostate

to 2009 , there was an increased incidence of emergency department visits associated with adverse events including catheterization ( $6.43 \%$ increase/year). In addition, a number of studies have highlighted the possible detrimental impact of conservative medical management of $\mathrm{BPH}$, leading to adverse events and medical treatment failure. ${ }^{16}$ That being said, the "gold standard" surgical treatment for BPH, TURP, is not an option for some patients because of the morbidity associated with the procedure. If such patients can be identified, it is plausible that an alternative surgical option can be applied, or alternatively, it can be determined that conservative management is the sole and best option.

Several of our findings are noteworthy. In this study, we demonstrate that the majority of patients $(65.2 \%)$ underwent TURP, a third of patients $(33.1 \%)$ underwent LVP, and the remaining patients $(1.7 \%)$ underwent LEP. This is representative of the surgical modality of choice for the treatment of BPH by urologists; most do not perform LEP because of its steep learning curve. ${ }^{17}$
Second, our comparative effectiveness assessment of the competing treatment modalities demonstrates the relative safety of all three surgical approaches. Specifically, no significant difference in overall complications or perioperative mortality between the three groups was identified. As well, no difference in postoperative renal function was identified between the three surgical groups. We corroborate the results of the meta-analysis by Ahyai and associates, ${ }^{18}$ which demonstrated similar morbidity and mortality between TURP and other minimally invasive BPH treatments. TURP patients in our study did have higher rates of pulmonary complications. This is likely because significantly more TURP patients underwent general anesthesia compared with other surgical modalities. Moreover, we demonstrate that LVP is associated with decreased blood transfusions, pLOS, and reintervention rates. These findings support previous investigations that reported an association between potassium titanyl phosphate laser and decreased length of stay and blood transfusions. ${ }^{19,20}$ Finally, patients 
Table 3D. Uni- and Multivariable Logistic Regression Analysis of Predictors of Need for Perioperative Mortality in 7359 Patients Undergoing Either Transurethral Resection of the Prostate or Tissue Vaporization or Enucleation for Management of Benign Prostatic Hyperplasia (National Surgical Quality Improvement Program Database 2006-2009)

\begin{tabular}{|c|c|c|c|c|}
\hline & \multicolumn{2}{|c|}{ Univariable analyses } & \multicolumn{2}{|c|}{ Multivariable analyses } \\
\hline & Odds ratio & $\mathrm{P}$ value & Odds ratio & $\mathrm{P}$ value \\
\hline Age at procedure & $1.13(1.06-1.19)$ & $<0.001$ & $1.10(1.00-1.19)$ & 0.01 \\
\hline $\begin{array}{l}\text { Race } \\
\text { Caucasian } \\
\text { Other }\end{array}$ & $\begin{array}{c}1(\text { Ref }) \\
0.66(0.25-1.75)\end{array}$ & 0.4 & - & \\
\hline $\begin{array}{l}\text { Smoking } \\
\text { Nonsmoker } \\
\text { Smoker }\end{array}$ & $\begin{array}{c}1(\text { Ref }) \\
1.03(0.31-3.44)\end{array}$ & 0.9 & - & \\
\hline $\begin{array}{l}\text { BMI } \\
<25 \\
25-30 \\
>30\end{array}$ & $\begin{array}{l}1(\text { Ref }) \\
0.16(0.05-0.48) \\
0.36(0.13-0.88)\end{array}$ & $\begin{array}{c}<0.001 \\
0.02\end{array}$ & $\begin{array}{l}1 \text { (Ref) } \\
0.29(0.06-1.41) \\
0.99(0.27-3.66)\end{array}$ & $\begin{array}{l}0.1 \\
0.9\end{array}$ \\
\hline $\begin{array}{l}\text { Preoperative comorbidities } \\
\text { Diabetes } \\
\text { Hypertension } \\
\text { Cardiovascular } \\
\text { Other }\end{array}$ & $\begin{array}{l}2.28(1.04-4.99) \\
2.03(0.81-4.97) \\
1.68(0.71-4.00) \\
5.57(2.60-11.92)\end{array}$ & $\begin{aligned} & 0.04 \\
& 0.1 \\
& 0.2 \\
< & 0.001\end{aligned}$ & 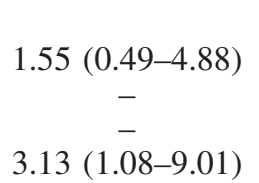 & 0.03 \\
\hline $\begin{array}{l}\text { Hematocrit }(\%) \\
<30 \\
30-45 \\
>45\end{array}$ & $\begin{array}{c}1(\text { Ref }) \\
0.09(0.01-0.23) \\
0.03(0.01-0.21)\end{array}$ & $\begin{array}{r}0.001 \\
<0.001\end{array}$ & $\begin{aligned} & 1(\text { Ref }) \\
& 0.21(0.07-0.66) \\
& 0.00(0.00-0.00)\end{aligned}$ & $\begin{array}{l}0.01 \\
0.9\end{array}$ \\
\hline $\begin{array}{l}\text { Anesthesia } \\
\text { General } \\
\text { Spinal }\end{array}$ & $\begin{array}{c}1 \text { (Ref) } \\
0.94(0.40-2.24)\end{array}$ & 0.9 & - & \\
\hline $\begin{array}{l}\text { Surgery } \\
\text { TURP } \\
\text { Vaporization } \\
\text { Enucleation }\end{array}$ & $\begin{array}{l}1(\text { Ref }) \\
0.68(0.29-1.62) \\
0.00(0.00-0.00)\end{array}$ & $\begin{array}{l}0.4 \\
0.9\end{array}$ & $\begin{array}{l}1(\text { Ref }) \\
1.29(0.44-3.78) \\
0.00(0.00-0.00)\end{array}$ & 0.9 \\
\hline $\begin{array}{l}\text { Preoperative albumin }(\mathrm{g} / \mathrm{dL}) \\
<2.5 \\
2.5-3.5 \\
>3.5 \\
\text { Operative time }\end{array}$ & $\begin{array}{l}1(\text { Ref }) \\
0.15(0.05-0.48) \\
0.03(0.01-0.11) \\
0.98(0.97-1.00)\end{array}$ & $\begin{aligned} & 0.001 \\
< & 0.001 \\
& 0.1\end{aligned}$ & $\begin{array}{c}1 \text { (Ref) } \\
0.29(0.08-1.03) \\
0.13(0.03-0.59) \\
-\end{array}$ & $\begin{array}{l}0.06 \\
0.01\end{array}$ \\
\hline
\end{tabular}

$\mathrm{BMI}=$ body mass index; TURP $=$ transurethral resection of the prostate.

undergoing LEP had decreased pLOS. Taken together, our findings suggest that LVP and LEP may be considered as an alternative treatment modality in patients who are at risk of adverse events after BPH surgery.

Our analyses reveal important findings with regard to preoperative risk evaluation. For example, age was an independent predictor of three primary end points-namely, overall complications, perioperative mortality, and pLOS. A study by Cullen and associates ${ }^{21}$ corroborates these results in patients undergoing TURP. They found increasing age to be associated with increased length of stay and complication rates. In addition, Jeldres and coworkers ${ }^{22}$ demonstrated that age was a significant independent predictor of 30-day mortality after TURP. Similar to age, non-Caucasian race was an independent predictor of complications after BPH surgery. While patients of non-Caucasian races often present with more comorbidities, ${ }^{23}$ race was nevertheless an independent predictor of overall complications. These findings confirm results for other medical conditions, where race predicted the risk of morbidity and mortality after surgery. ${ }^{24}$ While biologic hypotheses may be advanced, a worrisome body of literature has demonstrated that non-Caucasian patients may not have access to the best care. ${ }^{25}$ Taken together, these findings provide important information to guide patient counseling and decision making.

With regard to serum markers, our study demonstrates the importance of serum albumin levels for prediction of adverse events after BPH surgery. Specifically, normal preoperative levels of albumin were associated with a decreased rate of blood transfusions, pLOS, overall complications, and, most importantly, a decrease in perioperative mortality. Indeed, albumin is correlated with nutritional status and is often lowered in chronic disease states. ${ }^{26}$ Therefore, in patients undergoing BPH surgery, hypoalbuminemia may represent a poor constitutional state, which would portend increased risk for perioperative morbidity and mortality. The relationship between albumin and all cause mortality was previously reported by Goldwasser and colleagues, ${ }^{27}$ who examined 
Table 3E. Uni- and Multivariable Logistic Regression Analysis of Predictors of Need for Re-Intervention in 7359 Patients Undergoing Either Transurethral Resection of the Prostate or Tissue Vaporization or Enucleation for Management of Benign Prostatic Hyperplasia (National Surgical Quality Improvement Program Database 2006-2009)

\begin{tabular}{|c|c|c|c|c|}
\hline & Univaria & & Multivaric & \\
\hline & Odds ratio & $\mathrm{P}$ value & Odds ratio & $\mathrm{P}$ value \\
\hline Age at procedure & $1.02(1.00-1.04)$ & 0.03 & $1.01(0.99-1.02)$ & 0.06 \\
\hline $\begin{array}{l}\text { Race } \\
\text { Caucasian } \\
\text { Other }\end{array}$ & $\begin{array}{c}1(\text { Ref }) \\
1.26(0.85-1.78)\end{array}$ & 0.3 & - & \\
\hline $\begin{array}{l}\text { Smoking } \\
\text { Nonsmoker } \\
\text { Smoker }\end{array}$ & $\begin{array}{c}1(\text { Ref }) \\
1.08(0.58-1.73)\end{array}$ & 0.9 & - & \\
\hline $\begin{array}{l}\text { BMI } \\
<25 \\
25-30 \\
>30\end{array}$ & $\begin{array}{l}1 \text { (Ref) } \\
0.89(0.60-1.32) \\
0.74(0.49-1.19)\end{array}$ & $\begin{array}{l}0.5 \\
0.3\end{array}$ & - & \\
\hline $\begin{array}{l}\text { Preoperative comorbidities } \\
\text { Diabetes } \\
\text { Hypertension } \\
\text { Cardiovascular } \\
\text { Other }\end{array}$ & $\begin{array}{l}0.76(0.49-1.21) \\
0.85(0.61-1.21) \\
1.01(0.95-1.58) \\
0.93(0.59-1.44)\end{array}$ & $\begin{array}{l}0.2 \\
0.4 \\
0.9 \\
0.7\end{array}$ & $\begin{array}{l}- \\
- \\
- \\
-\end{array}$ & \\
\hline $\begin{array}{l}\text { Hematocrit }(\%) \\
<30 \\
30-45 \\
>45\end{array}$ & $\begin{array}{c}1 \text { (Ref }) \\
0.36(0.20-0.64) \\
0.45(0.23-0.89)\end{array}$ & $\begin{array}{c}<0.001 \\
0.02\end{array}$ & $\begin{array}{c}1 \text { (Ref) } \\
0.62(0.42-1.02) \\
0.53(0.27-1.07)\end{array}$ & $\begin{array}{l}0.08 \\
0.07\end{array}$ \\
\hline $\begin{array}{l}\text { Anesthesia } \\
\text { General } \\
\text { Spinal }\end{array}$ & $\begin{array}{c}1 \text { (Ref) } \\
0.99(0.68-1.45)\end{array}$ & 0.9 & - & \\
\hline $\begin{array}{l}\text { Surgery } \\
\text { TURP } \\
\text { Vaporization } \\
\text { Enucleation }\end{array}$ & $\begin{array}{c}1 \text { (Ref) } \\
0.60(0.40-0.89) \\
0.72(0.17-2.29)\end{array}$ & $\begin{array}{l}0.01 \\
0.7\end{array}$ & $\begin{array}{c}1(\text { Ref }) \\
0.63(0.42-0.93) \\
0.78(0.19-3.23)\end{array}$ & $\begin{array}{l}0.02 \\
0.7\end{array}$ \\
\hline $\begin{array}{l}\text { Preoperative albumin }(\mathrm{g} / \mathrm{dL}) \\
<2.5 \\
2.5-3.5 \\
>3.5 \\
\text { Operative time }\end{array}$ & $\begin{array}{c}1 \text { (Ref) } \\
0.46(0.16-1.30) \\
0.40(0.15-1.04) \\
1.00(1.00-1.06)\end{array}$ & $\begin{array}{l}0.1 \\
0.06 \\
0.1\end{array}$ & - & \\
\hline
\end{tabular}

$\mathrm{BMI}=$ body mass index; TURP $=$ transurethral resection of the prostate.

numerous large population cohort studies and demonstrated a direct relationship in adjusted analyses between albumin and decreased mortality. Lambert and associates ${ }^{28}$ also reported an increase in overall complications, in overall mortality and in cancer-specific mortality after cystectomy in patients with low levels of albumin.

Another important finding is that a preoperative hematocrit of $30 \%$ or more is associated with decreased rates of blood transfusions and pLOS. While the decreased need for blood transfusions may be easy to explain, we also show that a higher hematocrit is associated with a decrease in perioperative mortality. Previous investigations have demonstrated that low hematocrit is associated with chronic inflammatory disease states and infectious processes and has also been shown to increase mortality in patients undergoing hemodialysis. ${ }^{29}$ Therefore, it is possible that patients with $\mathrm{BPH}$ and low hematocrit have a combination of chronic prostatic inflammation and lower hemoglobin, which may place them at risk of perioperative mortality.
Among its many positives, the NSQIP database also has several drawbacks. First, the lack of hospital and payer characteristics prevented assessment of the impact of hospital volume or other socioeconomic factors such as insurance status on perioperative outcomes. In addition, the voluntary participation in the NSQIP requires resources, which may select for larger, high-volume, and academic institutions. As such, our results may not be generalizable to the broad medical community.

Second, an important consideration is that identification of cases for this study was based on CPT codes. Therefore, this study has limitations inherent to the use of CPT coding (such as coding errors). In addition, more accurate details were not available concerning the type of surgery performed. As such, it was not clear whether prostatic laser vaporization was performed by PVP or by holmium laser. Moreover, with regards to PVP, during this study period, only the HPS $120 \mathrm{~W}$ system was available in North America. As such, outcomes related to the use of the current 
180W system cannot be assessed. ${ }^{30}$ Also, while LEP was most likely performed using the holmium laser system, it may also have been performed with other laser modalities such as thulium and GreenLight, ${ }^{31,32}$ and also the small number of LEP procedures performed during the study period might represent another limitation. As well, it is unknown what type of TURP was performed-mono $v s$ bipolar.

Another limitation of this study was the lack of preoperative prostate gland information including serum prostatespecific antigen level and prostate gland size. Furthermore, patient use of anticoagulants was not available, which could have a significant impact on postoperative outcomesnamely, the need for blood transfusions. Moreover, serum albumin values were only available for $58 \%$ of our patient population. Therefore, the importance of albumin as a preoperative predictor of surgical morbidity needs to be further evaluated before it can be used in this context.

Finally, it is possible that with the emergence of LVP in recent years, our data may not provide an accurate depiction of the most contemporary trends in the surgical treatment of patients with $\mathrm{BPH}$.

\section{Conclusions}

In our analysis of data from the NSQIP initiative, all three major surgical modalities for treatment of patients with $\mathrm{BPH}$ were found to be safe. Age and race were independent predictors of adverse outcomes after BPH surgery. In patients with these attributes, preoperative surgical risk evaluation is warranted. Finally, preoperative hematocrit and albumin levels represent reliable serum markers for prediction of adverse outcomes, suggesting that these markers should be evaluated before BPH surgery.

\section{Disclosure Statement}

No competing financial interests exist.

\section{References}

1. Garraway WM, Collins GN, Lee RJ. High prevalence of benign prostatic hypertrophy in the community. Lancet 1991;338:469-471.

2. Berges RR, Pientka L. Management of the BPH syndrome in Germany: Who is treated and how? Eur Urol 1999; 36(suppl 3):21-27.

3. Norman RW, Nickel JC, Fish D, Pickett SN. 'Prostaterelated symptoms' in Canadian men 50 years of age or older: Prevalence and relationships among symptoms. $\underline{\mathrm{Br} J}$ Urol 1994;74:542-550.

4. Lee E, Yoo KY, Kim Y, et al. Prevalence of lower urinary tract symptoms in Korean men in a community-based study. Eur Urol 1998;33:17-21.

5. Roghmann F, Ghani KR, Kowalczyk KJ, et al. Incidence and treatment patterns in males presenting with lower urinary tract symptoms to the emergency department in the United States. J Urol 2013;190:1798-1804.

6. Groves HK, Chang D, Palazzi K, et al. The incidence of acute urinary retention secondary to BPH is increasing among California men. Prostate Cancer Prostatic Dis 2013; 16:260-265.

7. Oelke M, Bachmann A, Descazeaud A, et al. EAU guidelines on the treatment and follow-up of non-neurogenic male lower urinary tract symptoms including benign prostatic obstruction. Eur Urol 2013;64:118-140.

8. Madersbacher S, Alivizatos G, Nordling J, et al. EAU 2004 guidelines on assessment, therapy and follow-up of men with lower urinary tract symptoms suggestive of benign prostatic obstruction (BPH guidelines). Eur Urol 2004;46: 547-554.

9. Rassweiler J, Teber D, Kuntz R, Hofmann R. Complications of transurethral resection of the prostate (TURP) incidence, management, and prevention. Eur Urol 2006; 50:969-980.

10. Reich O, Gratzke C, Bachmann A, et al. Morbidity, mortality and early outcome of transurethral resection of the prostate: A prospective multicenter evaluation of 10,654 patients. J Urol 2008;180:246-249.

11. Herrmann TR, Liatsikos EN, Nagele U, et al. EAU guidelines on laser technologies. Eur Urol 2012;61:783795 .

12. Krambeck AE, Handa SE, and Lingeman JE. Experience with more than 1,000 holmium laser prostate enucleations for benign prostatic hyperplasia. J Urol 2013;189:S141S145.

13. Elmansy HM, Kotb A, Elhilali MM. Holmium laser enucleation of the prostate: Long-term durability of clinical outcomes and complication rates during 10 years of followup. J Urol 2011;186:1972-1976.

14. Andersson SO, Rashidkhani B, Karlberg L, et al. Prevalence of lower urinary tract symptoms in men aged 45 79 years: A population-based study of 40000 Swedish men. BJU Int 2004;94:327-331.

15. Coyne KS, Sexton CC, Thompson CL, et al. The prevalence of lower urinary tract symptoms (LUTS) in the USA, the UK and Sweden: Results from the Epidemiology of LUTS (EpiLUTS) study. BJU Int 2009;104:352-360.

16. Stroup SP, Palazzi-Churas K, Kopp RP, Parsons JK. Trends in adverse events of benign prostatic hyperplasia (BPH) in the USA, 1998 to 2008. BJU Int 2012;109: 84-87.

17. El-Hakim A, Elhilali MM. Holmium laser enucleation of the prostate can be taught: The first learning experience. BJU Int 2002;90:863-869.

18. Ahyai SA, Gilling P, Kaplan SA, et al. Meta-analysis of functional outcomes and complications following transurethral procedures for lower urinary tract symptoms resulting from benign prostatic enlargement. Eur Urol 2010;58:384397.

19. Alivizatos G, Skolarikos A, Chalikopoulos D, et al. Transurethral photoselective vaporization versus transvesical open enucleation for prostatic adenomas $>80 \mathrm{ml}$ : 12-mo results of a randomized prospective study. Eur Urol 2008;54:427-437.

20. Bouchier-Hayes DM, Anderson P, Van Appledorn S, et al. KTP laser versus transurethral resection: Early results of a randomized trial. J Endourol 2006;20:580-585.

21. Cullen DJ, Apolone G, Greenfield S, et al. ASA Physical Status and age predict morbidity after three surgical procedures. Ann Surg 1994;220:3-9.

22. Jeldres $\mathrm{C}$, Isbarn $\mathrm{H}$, Capitanio U, et al. Development and external validation of a highly accurate nomogram for the prediction of perioperative mortality after transurethral resection of the prostate for benign prostatic hyperplasia. J Urol 2009; 182:626-632.

23. Frierson GM, Howard EN, DeFina LE, et al. Effect of race and socioeconomic status on cardiovascular risk factor 
burden: The Cooper Center Longitudinal Study. Ethn Dis 2013;23:35-42.

24. Sammon J, Trinh VQ, Ravi P, et al. Health care-associated infections after major cancer surgery: Temporal trends, patterns of care, and effect on mortality. Cancer 2013;119: 2317-2324.

25. Trinh QD, Sun M, Sammon J, et al. Disparities in access to care at high-volume institutions for uro-oncologic procedures. Cancer 2012;118:4421-4426.

26. Goldwasser P. Albumin trend and mortality. Am J Kidney Dis 1997;30:308-309.

27. Goldwasser P, Feldman J. Association of serum albumin and mortality risk. J Clin Epidemiol 1997 50:693-703.

28. Lambert JW, Ingham M, Gibbs BB, et al. Using preoperative albumin levels as a surrogate marker for outcomes after radical cystectomy for bladder cancer. Urology 2013; 81:587-592.

29. Ma JZ, Ebben J, Xia H, Collins AJ. Hematocrit level and associated mortality in hemodialysis patients. J Am Soc Nephrol 1999;10:610-619.

30. Bachmann A, Muir GH, Collins EJ, et al. 180-W XPS GreenLight laser therapy for benign prostate hyperplasia: Early safety, efficacy, and perioperative outcome after 201 procedures. Eur Urol 2012;61:600-607.

31. Ben-Zvi T, Hueber PA, Abdollah F, et al. Short term outcomes of GreenLight vapor incision technique (VIT) of the prostate: Comparison of outcomes to standard GreenLight $120 \mathrm{~W}$ HPS vaporization in prostate volumes greater than 80 cc. Can J Urol 2013;20:6633-6639.

32. Iacono F, Prezioso D, Di Lauro G, et al. Efficacy and safety profile of a novel technique, ThuLEP (Thulium laser enu- cleation of the prostate) for the treatment of benign prostate hypertrophy. Our experience on 148 patients. BMC Surg 2012;12(suppl 1): S21.

Address correspondence to: Quoc-Dien Trinh, MD Brigham and Women's Hospital Harvard Medical School 45 Francis Street, ASB II-3 Boston, MA 02115

E-mail: trinh.qd@gmail.com

\begin{aligned} & \multicolumn{1}{c|}{ Abbreviations Used } \\ & $\mathrm{ACS}=$ American College of Surgeons \\ & $\mathrm{BMI}=$ body mass index \\ & $\mathrm{BPH}=$ benign prostatic hyperplasia \\ & HoLEP $=$ holmium laser enucleation of the prostate \\ & $\mathrm{IPSS}=$ International Prostate Symptom Score \\ & $\mathrm{LEP}=$ laser enucleation of the prostate \\ & $\mathrm{LUTS}=$ lower urinary tract symptoms \\ & $\mathrm{LVP}=$ laser vaporization of the prostate \\ & $\mathrm{NSQIP}=$ National Surgical Quality Improvement Program \\ & $\mathrm{OR}=$ odds ratio \\ & pLOS $=$ prolonged length of stay \\ & TURP $=$ transurethral resection of the prostate \\ & $\mathrm{UR}=$ urinary retention \end{aligned}




\section{This article has been cited by:}

1. Jae Hung Jung, Tae Young Shin, Karen Ann McCutcheon, Michael Borofsky, Vikram Narayan, Shamar Young, Jafar Golzarian, Myung Ha Kim, Balaji Reddy, Philipp Dahm. 2017. Prostatic arterial embolization for the treatment of lower urinary tract symptoms in men with benign prostatic hyperplasia. Cocbrane Database of Systematic Reviews 58. . [Crossref]

2. Jae Hung Jung, Karen Ann McCutcheon, Balaji Reddy, Michael Borofsky, Vikram Narayan, Myung Ha Kim, Philipp Dahm. 2017. Prostatic urethral lift for the treatment of lower urinary tract symptoms in men with benign prostatic hyperplasia. Cocbrane Database of Systematic Reviews 65. . [Crossref]

3. Xia Leilei, Taylor Benjamin L., Pulido Jose E., Mucksavage Phillip, Lee David I., Guzzo Thomas J.. 2017. Predischarge Predictors of Readmissions and Postdischarge Complications in Robot-Assisted Radical Prostatectomy. Journal of Endourology 31:9, 864-871. [Abstract] [Full Text HTML] [Full Text PDF] [Full Text PDF with Links]

4. Akshay Sood, Naveen Kachroo, Firas Abdollah, Jesse D. Sammon, Björn Löppenberg, Tarun Jindal, Maxine Sun, Quoc-Dien Trinh, Mani Menon, James O. Peabody. 2017. An Evaluation of the Timing of Surgical Complications Following Radical Cystectomy: Data From the American College of Surgeons National Surgical Quality Improvement Program. Urology 103, 91-98. [Crossref]

5. S. Duke Herrell. Robotic Surgery: Past, Present, and Future 459-472. [Crossref]

6. Simon Allen, Ivan Gerasimovich Aghajanyan. 2016. Thermobalancing conservative treatment for moderate-to-low-degree lower urinary tract symptoms (LUTS) secondary to prostate enlargement. Cogent Medicine 3:1. . [Crossref]

7. C Gilfrich, H Leicht, C Fahlenbrach, E Jeschke, G Popken, J U Stolzenburg, L Weißbach, C Zastrow, C Günster. 2016. Morbidity and mortality after surgery for lower urinary tract symptoms: a study of 95577 cases from a nationwide German health insurance database. Prostate Cancer and Prostatic Diseases 19:4, 406-411. [Crossref]

8. Christian P. Meyer, Arturo J. Rios-Diaz, Deepansh Dalela, Praful Ravi, Akshay Sood, Julian Hanske, Felix K.H. Chun, Adam S. Kibel, Stuart R. Lipsitz, Maxine Sun, Quoc-Dien Trinh. 2016. The association of hypoalbuminemia with early perioperative outcomes - A comprehensive assessment across 16 major procedures. The American Journal of Surgery . [Crossref]

9. Mitchell Christopher R., Hendrick Richard J., Webster Robert J. III, Herrell S. Duke. 2016. Toward Improving Transurethral Prostate Surgery: Development and Initial Experiments with a Prototype Concentric Tube Robotic Platform. Journal of Endourology 30:6, 692-696. [Abstract] [Full Text HTML] [Full Text PDF] [Full Text PDF with Links]

10. Marianne Schmid, H. Abraham Chiang, Akshay Sood, Logan Campbell, Felix K.-H. Chun, Deepansh Dalela, James Okwara, Jesse D. Sammon, Adam S. Kibel, Mani Menon, Margit Fisch, Quoc-Dien Trinh. 2016. Causes of hospital readmissions after urologic cancer surgery. Urologic Oncology: Seminars and Original Investigations 34:5, 236.e1-236.e11. [Crossref]

11. Akshay Sood, Kaustav Majumder, Naveen Kachroo, Jesse D. Sammon, Firas Abdollah, Marianne Schmid, Linda Hsu, Wooju Jeong, Christian P. Meyer, Julian Hanske, Richard Kalu, Mani Menon, Quoc-Dien Trinh. 2016. Adverse Event Rates, Timing of Complications, and the Impact of Specialty on Outcomes Following Adrenal Surgery: An Analysis of 30-Day Outcome Data From the American College of Surgeons National Surgical Quality Improvement Program (ACS-NSQIP). Urology 90, 62-68. [Crossref]

12. Akshay Sood, Firas Abdollah, Jesse D. Sammon, Victor Kapoor, Craig G. Rogers, Wooju Jeong, Dane E. Klett, Julian Hanske, Christian P. Meyer, James O. Peabody, Mani Menon, Quoc-Dien Trinh. 2015. An evaluation of the timing of surgical complications following nephrectomy: data from the American College of Surgeons National Surgical Quality Improvement Program (ACS-NSQIP). World Journal of Urology 33:12, 2031-2038. [Crossref]

13. Julian Hanske, Alejandro Sanchez, Marianne Schmid, Christian P. Meyer, Firas Abdollah, Florian Roghmann, Adam S. Feldman, Adam S. Kibel, Jesse D. Sammon, Joachim Noldus, Quoc-Dien Trinh, Jairam R. Eswara. 2015. Comparison of 30-day perioperative outcomes in adults undergoing open versus minimally invasive pyeloplasty for ureteropelvic junction obstruction: analysis of 593 patients in a prospective national database. World Journal of Urology 33:12, 2107-2113. [Crossref]

14. Praful Ravi, Akshay Sood, Marianne Schmid, Firas Abdollah, Jesse D. Sammon, Maxine Sun, Dane E. Klett, Briony Varda, James O. Peabody, Mani Menon, Adam S. Kibel, Paul L. Nguyen, Quoc-Dien Trinh. 2015. Racial/Ethnic Disparities in Perioperative Outcomes of Major Procedures. Annals of Surgery 262:6, 955-964. [Crossref]

15. Akshay Sood, Firas Abdollah, Jesse D. Sammon, Kaustav Majumder, Marianne Schmid, James O. Peabody, Mark A. Preston, Adam S. Kibel, Mani Menon, Quoc-Dien Trinh. 2015. The Effect of Body Mass Index on Perioperative Outcomes After Major Surgery: Results from the National Surgical Quality Improvement Program (ACS-NSQIP) 2005-2011. World Journal of Surgery 39:10, 2376-2385. [Crossref]

16. Joseph J. Pariser, Shane M. Pearce, Sanjay G. Patel, Gregory T. Bales. 2015. Reply. Urology 86:4, 726. [Crossref]

17. V. Misrä̈, N. Barry Delongchamps, S. Lebdai, A.R. Azzouzi, A. Benchikh, J.N. Cornu, O. Dumonceau, M. Fourmarier, O. Haillot, B. Lukacs, R. Mathieu, G. Robert, A. de La Taille, A. Descazeaud. 2015. Complications graves et inattendues de la 
chirurgie de l'hyperplasie bénigne de prostate : résultats de l'enquête du CTMH auprès des urologues de l'AFU. Progrès en Urologie 25:10, 583-589. [Crossref]

18. Marianne Schmid, Akshay Sood, Logan Campbell, Victor Kapoor, Deepansh Dalela, Dane E. Klett, Felix K.-H. Chun, Adam S. Kibel, Jesse D. Sammon, Mani Menon, Margit Fisch, Quoc-Dien Trinh. 2015. Impact of smoking on perioperative outcomes after major surgery. The American Journal of Surgery 210:2, 221-229.e6. [Crossref]

19. Francesco Montorsi, Giorgio Gandaglia. 2015. Re: Riccardo Autorino, Homayoun Zagar, Mirandolino B. Mariano, et al. Perioperative Outcomes of Robotic and Laparoscopic Simple Prostatectomy: A European-American Multi-institutional Analysis. Eur Urol 2015;68:86-94Re: Matthew Bultitude, Ben Challacombe. Simple Prostatectomy: A Step Too Far for Laparoscopy? Eur Urol 2015;68:95-6. European Urology 68:1, e7-e8. [Crossref]

20. Chughtai Bilal I., Simma-Chiang Vannita, Lee Richard, Isaacs Abby, Te Alexis E., Kaplan Steven A., Sedrakyan Art. 2015. Trends and Utilization of Laser Prostatectomy in Ambulatory Surgical Procedures for the Treatment of Benign Prostatic Hyperplasia in New York State (2000-2011). Journal of Endourology 29:6, 700-706. [Abstract] [Full Text HTML] [Full Text PDF] [Full Text PDF with Links]

21. Sammy E. Elsamra, Nikhil Gupta, Haris Ahmed, David Leavitt, Jessica Kreshover, Louis Kavoussi, Lee Richstone. 2014. WITHDRAWN: Robotic assisted laparoscopic simple suprapubic prostatectomy - The Smith Institute for Urology experience with an evolving technique. Asian Journal of Urology . [Crossref]

22. Pariser Joseph J., Famakinwa Olufenwa J., Pearce Shane M., Chung Doreen E.. 2014. High-Power Thulium Laser Vaporization of the Prostate: Short-Term Outcomes of Safety and Effectiveness. Journal of Endourology 28:11, 1357-1362. [Abstract] [Full Text HTML] [Full Text PDF] [Full Text PDF with Links] [Supplemental Material]

23. Sammy E. Elsamra, Nikhil Gupta, Haris Ahmed, David Leavitt, Jessica Kreshover, Louis Kavoussi, Lee Richstone. 2014. Robotic assisted laparoscopic simple suprapubic prostatectomy - The Smith Institute for Urology experience with an evolving technique. Asian Journal of Urology 1:1, 55-59. [Crossref] 